\title{
Switching Loss Characteristics of Sequences \\ Involving Active State Division in Space Vector Based PWM
}

\author{
Di Zhao ${ }^{*}$, G. Narayanan ${ }^{* *}$ and Raja Ayyanar ${ }^{*}$ \\ *Department of Electrical Engineering \\ Arizona State University \\ Tempe, AZ 85287, USA \\ ${ }^{* *}$ Department of Electrical Engineering \\ Indian Institute of Science \\ Bangalore, India
}

\begin{abstract}
This paper analyzes the switching loss characteristics of sequences involving division of active state duration in space vector based PWM. This analysis, together with the THD performance of the different sequences, reported recently, is used to design new hybrid PWM techniques for induction motor drives, which result in simultaneous reduction in both THD as well as inverter switching losses. Experimental results are presented to demonstrate the feasibility and advantages of the proposed PWM techniques.
\end{abstract}

Key words: PWM, space vector, hybrid PWM, switching loss, current ripple.

\section{INTRODUCTION}

Two popular approaches to real-time PWM generation are the Triangle Comparison (TC) approach and the Space Vector (SV) approach [1-3]. A significant, additional degree of freedom in space vector based PWM for three-phase, twolevel converters, compared to triangle comparison based PWM, is the division of active state time, and the choice in sequence of application of the different states in a sub-cycle [4-6]. This freedom can be exploited to achieve reduction in the harmonic distortion (THD) in line currents and/or to reduce the switching losses in the inverter. The effect of different sequences on THD has been studied in [4,6], and a hybrid space vector PWM technique using sequences that involve active state division, in conjunction with the conventional sequence, has been proposed to reduce THD.

The main objective of the present paper is to analyze the switching loss characteristics of the different sequences, which can be used to formulate new hybrid PWM techniques that result in simultaneous reduction in both THD and switching losses.

\section{BACKGROUND}

\section{A. $\quad$ Various switching sequences}

This work was funded by the Office of Naval Research (ONR) under contract N00014-02-01-0751

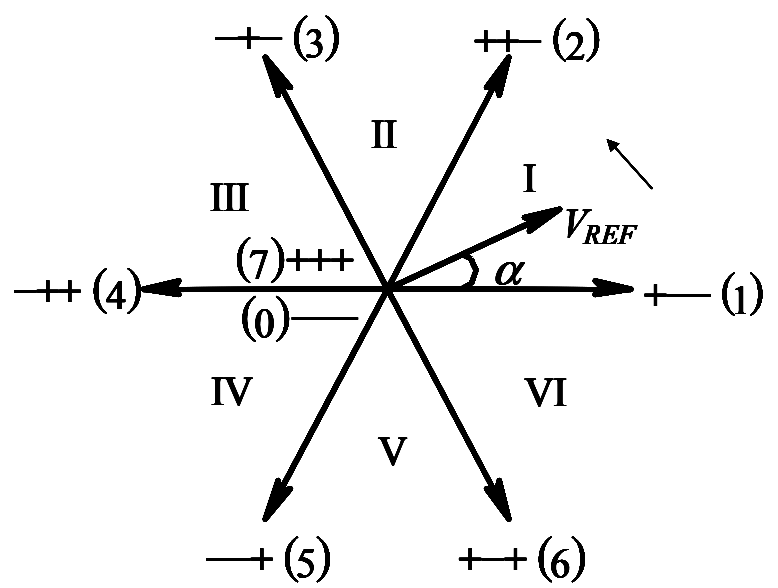

Fig.1 Inverter states and voltage vectors of three-phase inverter

Fig. 1 shows the voltage vectors produced by a threephase inverter, with the six active vectors dividing the space vector plane into six sectors. In space vector PWM (SVPWM), the desired reference vector is generated by time averaging the suitable discrete voltage vectors in every subcycle or sampling period $\mathrm{T}_{\mathrm{S}}$. For example, the reference vector $V_{R E F}$ in Sector I, as shown in Fig. 1, is generated by applying the active state 1 , the active state 2 and the zero states 0 and/or 7 for durations $T_{1}, T_{2}$ and $T_{Z}$ respectively [15]. For a given reference vector and $T s$, the duration $T_{1}, T_{2}$ and $\mathrm{T}_{\mathrm{Z}}$ are unique. However, each of these durations can be divided and applied in more than one distinct interval in a sub-cycle. There is also a choice in the sequence in which each of these states is applied [5,6].

Utilizing the freedom of active state division, seven different sequences are possible under the constraints that the total number of switchings in a sub-cycle is less than or equal to three (as in conventional SVPWM), and only one phase switches during a transition from one state to the next. These seven sequences are illustrated in Fig. 2. Since the six sectors are symmetric, the discussion is limited to Sector I alone. Fig. 2 shows the sequence corresponding to sector I only. 
(a)
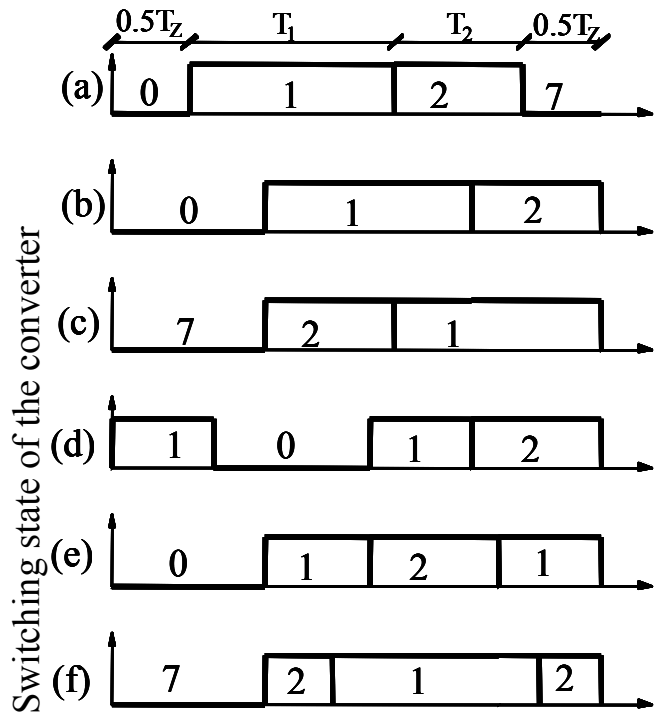

(g)

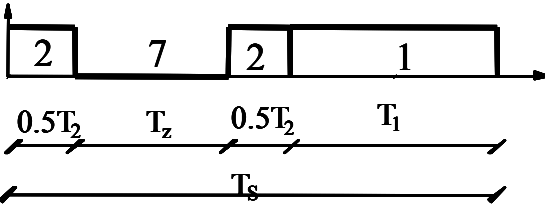

Fig. 2. Different switching sequences in Sector I

Referring to Fig. 2, 0127 is the conventional sequence, which divides $T_{Z}$ equally between the two zero states, 0 and $7 ; 012$ and 721 , referred to as the bus-clamping sequences in the literature, apply only one zero state during entire $\mathrm{T}_{\mathrm{Z}}$. Sequences 1012, 0121, 7212, and 2721 involve division of active vector time. In a given sub-cycle, these sequences result in multiple switchings of a particular phase, while clamping a different phase. For example, 1012 applies the active state 1 in two distinct, equal intervals of duration $T_{1} / 2$ each, and uses only one zero state (0). This results in double switching of phase ' $a$ ', single switching of phase ' $b$ ' and clamping of phase ' $c$ ' to the negative bus, in a sub-cycle in Sector I. Unlike the conventional and bus-clamping sequences, the sequences involving division of active state duration do not have equivalence in triangle comparison based PWM $[5,3]$.

\section{B. Hybrid PWM techniques to reduce THD}

The effect of different sequences on THD in line currents has been analyzed in $[4,6]$ using the concept of stator flux ripple. Stator flux ripple is the time integral of the error between the reference vector and the instantaneous applied voltage vector, and is a measure of the ripple in the line currents $[4,6]$. Based on this study, hybrid PWM techniques that use different sequences within a sector have been developed, with the objective to minimize THD. This basically involves dividing a sector, or the $\alpha-V_{\text {REF }}$ plane into zones of superior performance (in terms of THD) for each of the sequences, considered. A hybrid technique using 0127, 7212 and 0121 , shown in Fig. 3a, has been proposed in [4]. A hybrid PWM technique involving all the seven possible sequences $0127,0121,7212,1012,2721,012$ and 721 , as shown in Fig. $3 \mathrm{~b}$ has been proposed in [6], which achieves a reduction of more than $40 \%$ in THD at maximum modulation index.
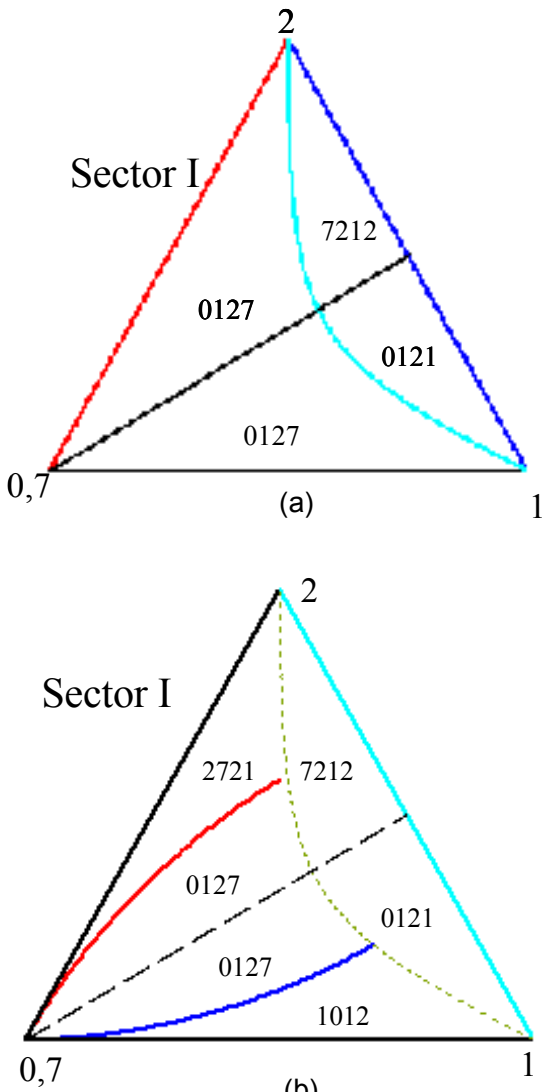

(b)

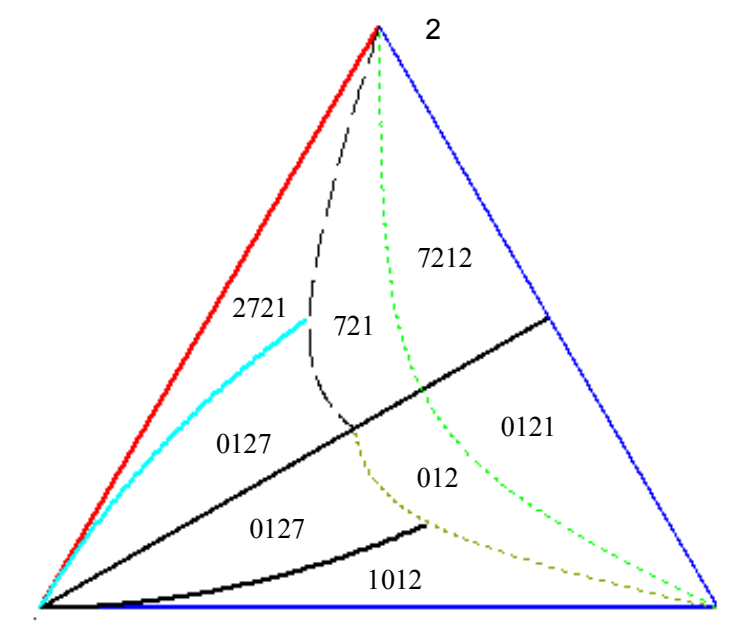

0,7

(c) 
The objective of this paper is to study the effect of the different sequences on the inverter switching losses, which can ultimately lead to development of hybrid PWM techniques that can achieve simultaneous reduction in both THD and switching loss.

\section{EFFECT OF SEQUENCES ON INVERTER SWITCHING LOSSES}

The switching loss in an IGBT depends mainly on the dc bus voltage, instantaneous line current, and turn-on and turnoff transition times. The dc bus voltage remains constant, and the transition times are also assumed to be constant for different instantaneous line currents. Hence, to compare the effects of different sequences on the switching loss in a given sub-cycle, it is enough to consider just the product of instantaneous line current magnitude of a particular phase and the number of switchings per sub-cycle in this phase, corresponding to the sequence considered. This product is referred to as the switching loss factor, Q. Since the three phases are symmetric, it is enough to analyze just one phase.
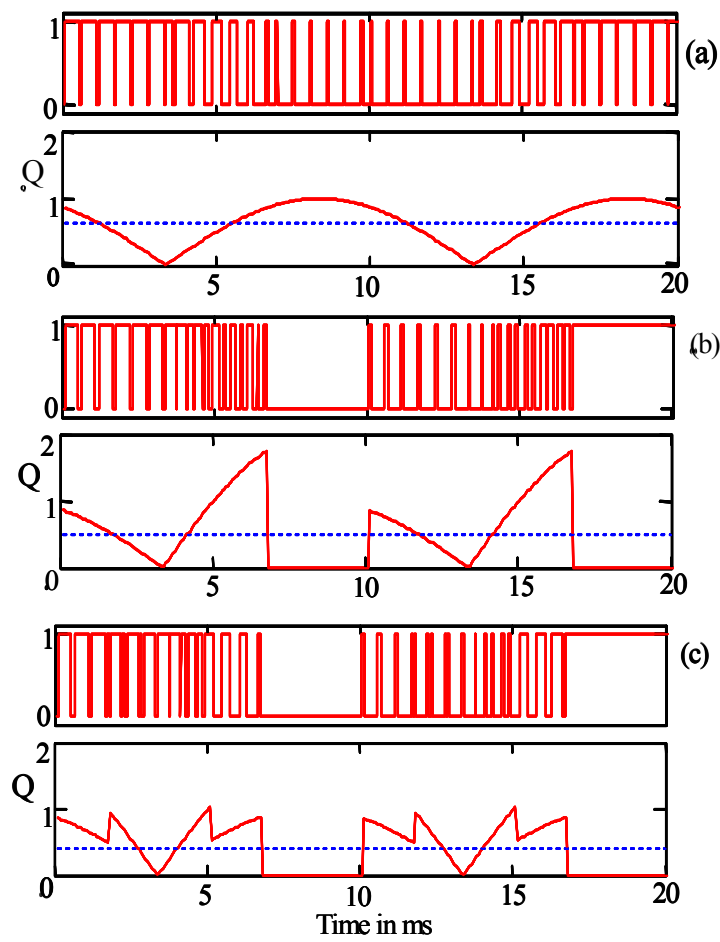

Fig. 4. PWM waveforms and normalized switching loss factor, Q, for different sequences. (a ) 0127, (b) 0121 and

(c) combination of 0121 and 1012 .

Fig. 4 shows the PWM waveform and the corresponding switching loss factor, Q over a complete fundamental period, for different types of sequences, corresponding to phase ' $a$ ' and at a power factor angle of $30^{\circ}$ leading. The average switching loss per phase over a fundamental period is also plotted. Since, the conventional sequence, 0127, always has just one switching per sub-cycle, the switching loss factor has identical waveform as that of the line current, as shown in Fig. 4a. Fig. 4b shows the PWM waveform and Q when sequence 0121 is applied throughout sector I (and sequences corresponding to 0121 in other sectors). This sequence results in single switching in sectors I and IV, double switching in sectors II and V and clamping in sectors III and VI. Hence, the switching loss factor is same as the instantaneous current in sectors I and IV, twice the instantaneous current in Sectors II and V and zero in sectors III and VI. Fig. 4c shows a possible hybrid switching scheme, where 0121 is used for $0<\alpha<30^{\circ}$ and 1012 for $30^{\circ}$ $<\alpha<60^{\circ}$ (and corresponding sequences in other sectors). This scheme clamps the phase when the current is at its peak value, thus resulting in significant reduction in the switching loss compared to that of conventional sequence.

To reduce switching losses, it is essential to ensure that the double switching occurs when the magnitude of the instantaneous line current is low, and clamping occurs when the current is near its peak value. For a given sequence, the sectors in which single or double switching or clamping occurs for a particular phase is fixed. Therefore, the switching loss factor depends mainly on the load power factor or the angle by which the line current lags/leads the line voltage. Also, it may be noted that the effect of different sequences on the switching loss does not depend on $V_{\text {REF }}$ or the fundamental frequency.

\section{A. Zones of superior performance in terms of switching losses}

Fig. 5 shows the total switching loss, $\mathrm{L}_{\mathrm{S}}$ (which is essentially the integral of the switching loss factor, Q over a fundamental cycle), for different sequences as a function of power factor angle. The switching loss is normalized with respect to the conventional sequence. As seen, different sequences perform better at different power factor angles, $\phi$. Hence, in order to design hybrid PWM techniques aimed at reducing switching loss, the $\alpha-\phi$ plane (instead of the $\alpha-V_{\text {REF }}$ plane as in THD study) needs to be partitioned into zones of superior performance for different sequences.

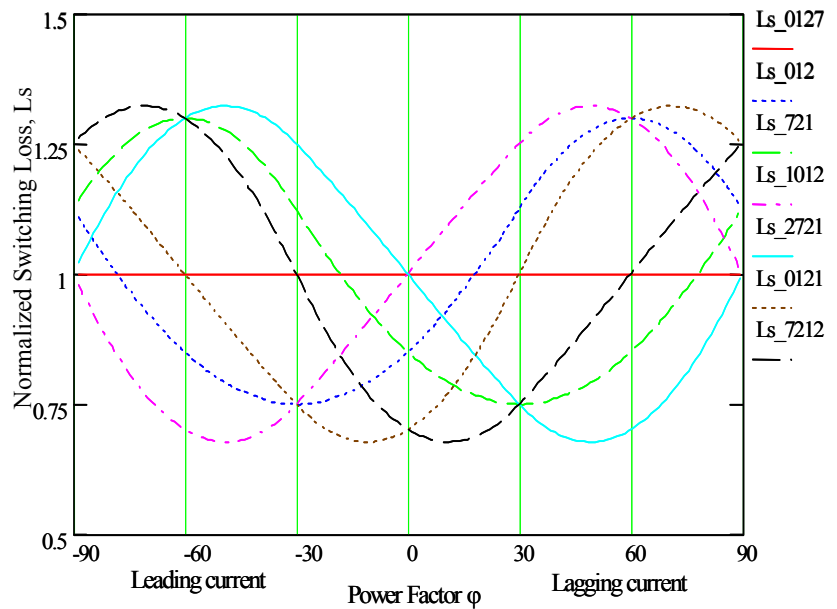

Fig. 5. Switching loss over a fundamental cycle for different sequences as a function of power factor angle 


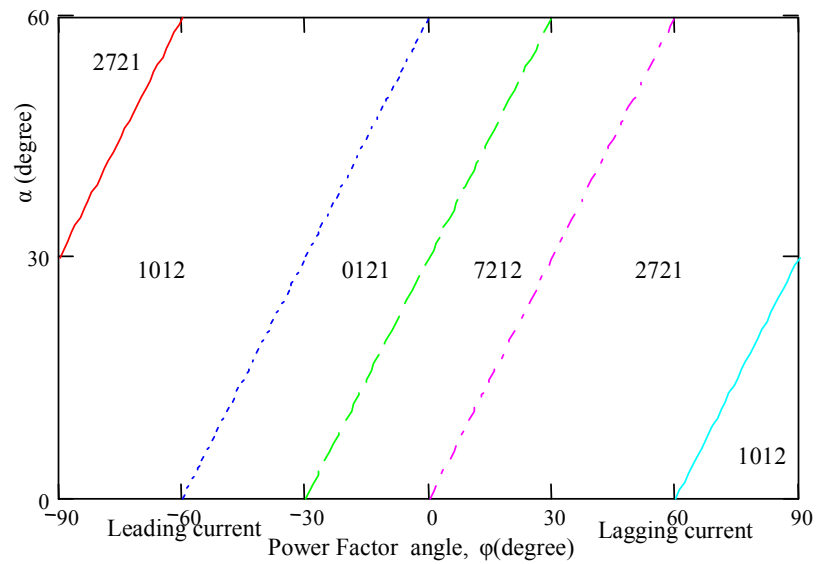

Fig. 6. Zones of superior performance for different sequences in terms of switching losses

Fig. 6 shows the hybrid PWM technique based solely on switching loss consideration, for the full range of power factor angles from $90^{\circ}$ leading to $90^{\circ}$ lagging. For example, at $\phi=30^{\circ}$, the hybrid scheme suggests using 2721 for $0<\alpha<$ $30^{\circ}$ and 7212 for $30^{\circ}<\alpha<60^{\circ}$ in sector I. The reduction in switching loss using the above switching-loss-optimized PWM technique is illustrated in Fig. 7. As seen, the switching loss optimized technique results in more than $30 \%$ reduction in switching loss compared to conventional SVPWM, over a wide range of power factor angles.

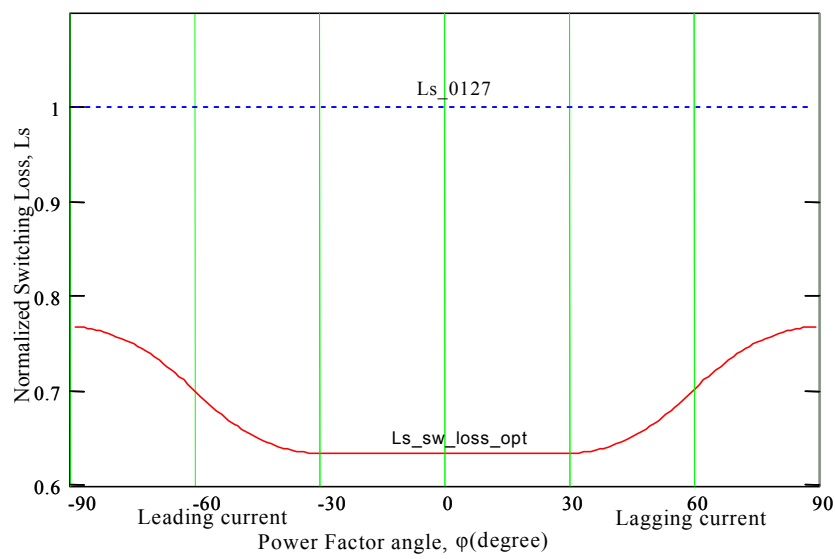

Fig.7. Reduction in switching loss using hybrid PWM given in Fig. 6, compared to conventional SVPWM.

It is obvious that a hybrid PWM technique optimized for switching loss reduction will not give optimum performance in terms of THD, and vice versa. Fig. 8 compares the performances of the THD optimized technique and the switching loss optimized technique along with the conventional space vector PWM. Fig. 8a shows the THD as a function of fundamental frequency, and Fig. $8 \mathrm{~b}$ shows the switching loss as a function of power factor angle, for the above three techniques. It is clear that neither of the techniques result in optimized performance if both THD and switching losses are to be minimized.
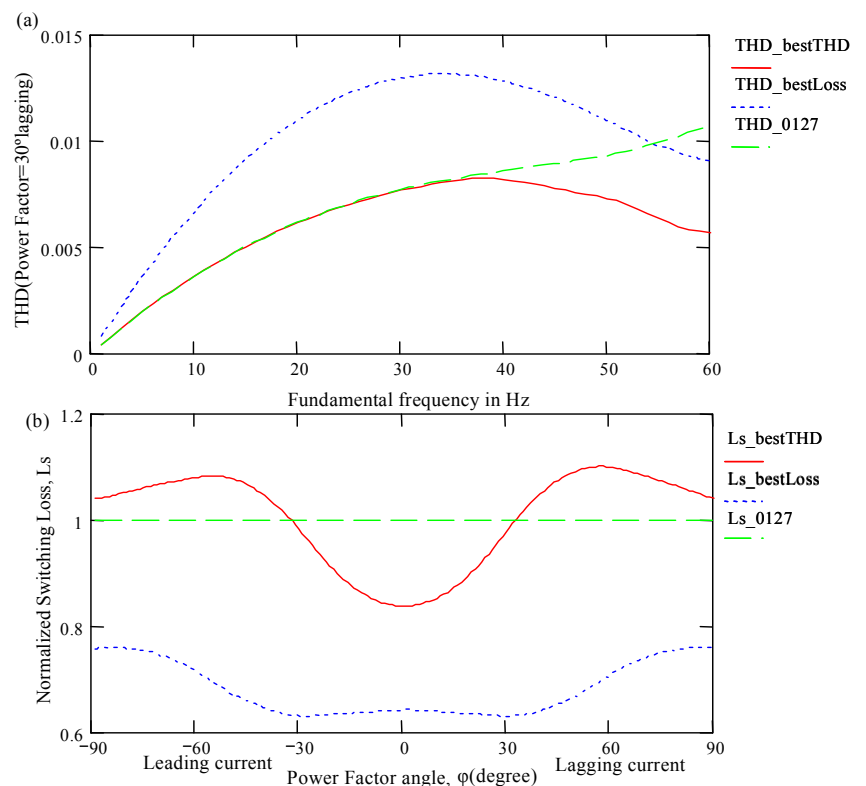

Fig. 8. Comparison of THD and switching loss performance of conventional PWM, THD optimized PWM and switching loss optimized PWM.

(a) THD vs. fundamental frequency (or Vref) at power factor angle of $30^{\circ}$ lagging and (b) switching loss vs. power factor angle

\section{B. Simultaneous reduction in THD and switching loss}

A possible approach to optimize both THD and switching loss performance is to define a performance index based on user defined weights to THD and switching losses respectively, and try to obtain the regions of superior performance for this performance factor in a threedimensional space $-\mathrm{V}_{\mathrm{REF}}, \alpha$ and $\phi$. However, it is unwieldy considering the wide range of $\mathrm{V}_{\mathrm{REF}}, \alpha$ and $\phi$, and almost impractical to implement using simple digital signal processors.

A more practical approach is to carefully study the THD characteristics and the switching loss characteristics of the different sequences, and identify zones of superior performance in the $\mathrm{V}_{\mathrm{REF}}-\alpha$ plane for a limited range of power factor angle typical of the application considered. A simple PWM technique that reduces both THD and switching losses for induction motor drive applications, where the typical power factor angles range from $20^{\circ}$ to $60^{\circ}$, is shown in Fig. 9.

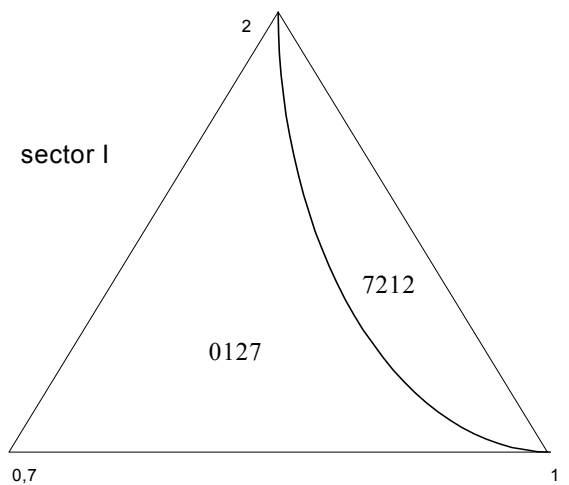

Fig. 9. A simple, hybrid PWM technique to simultaneously reduce both THD and switching loss in induction motor drives 
From Fig. 6 it can be seen that for the range of power factor between $20^{\circ}$ to $60^{\circ}$ lagging, 7212 is one of the sequences that result in minimum switching losses. Also, from Fig. 5, it can be seen that sequence 0121 results in higher switching loss than conventional sequence for $\phi>30^{\circ}$. The three-zone technique shown in Fig. 3, based on THD considerations, suggests use of 0121 for $0^{0}<\alpha<30^{\circ}$ and 7212 for $30^{\circ}<\alpha<60^{\circ}$ at higher values of $\mathrm{V}_{\text {REF }}$. However, the difference between the stator flux ripples of 0121 and 7212 is not very significant at high values of $\mathrm{V}_{\text {REF }}$. But, the sequence 7212 results in significantly lower switching loss than 0121 for typical power factor angles considered. From these observations, the three-zone hybrid PWM technique can be modified as shown in Fig. 9. The THD performance of the modified technique is close to that of the three-zone technique, but the switching loss is significantly reduced.
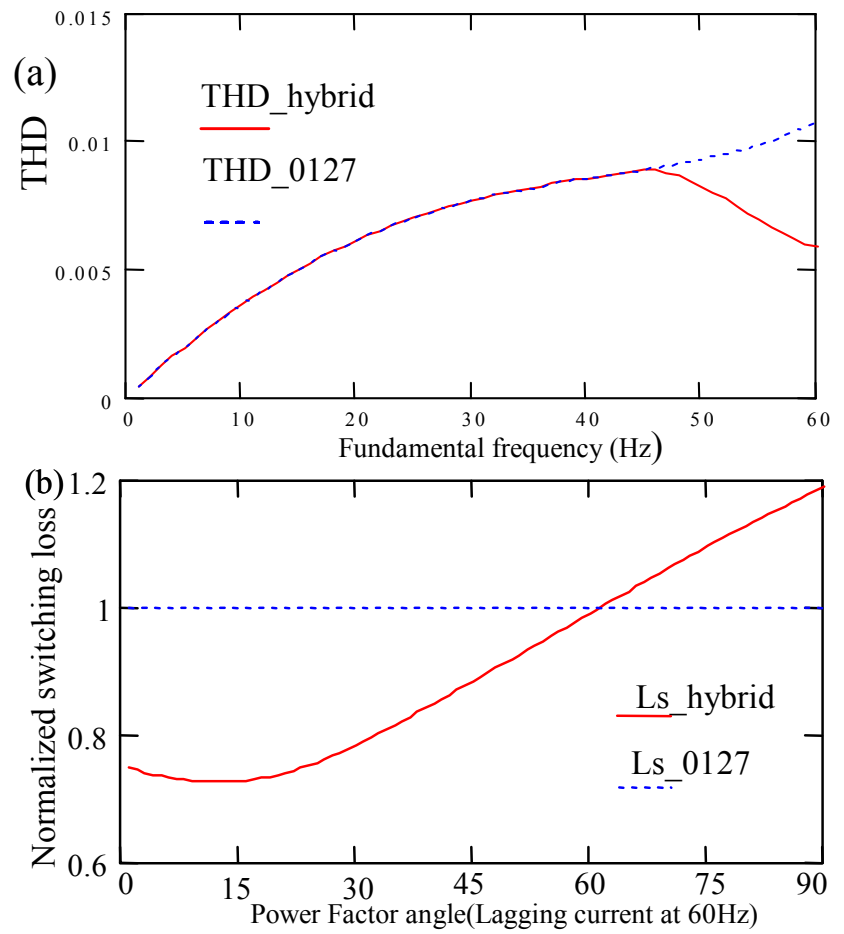

Fig. 10. THD and switching loss performance of combined hybrid PWM shown in Fig. 9, compared to conventional SVPWM

The THD obtained with this PWM technique, along with that of the conventional SVPWM is shown in Fig. 10a as a function of $\mathrm{V}_{\mathrm{REF}}$ (or fundamental frequency. Fig. 10b compares the switching losses obtained using the new PWM technique with that of conventional, as a function of power factor angle (corresponding to a fundamental frequency of 60 $\mathrm{Hz}$ ). As seen from these two figures, the proposed PWM technique achieves simultaneously a reduction of about $30 \%$ in THD and a reduction of more than $20 \%$ in switching loss under normal operating conditions - maximum $\mathrm{V}_{\mathrm{REF}}$ and a power factor angle of $30^{\circ}$, compared to conventional SVPWM.

Similarly, using the results shown in Fig. 5, the fivezone and seven-zone hybrid PWM techniques shown in Fig.3, which were obtained based solely on THD considerations, can also be modified to achieve simultaneous reduction in switching losses. Note that the resulting techniques do not require current feedback and are based on the assumption that the power factor angle for the application considered is between $20^{\circ}$ and $60^{\circ}$ lagging. The various hybrid PWM techniques to reduce both THD as well as switching losses are shown in Fig. 11. The scheme shown in Fig. 11a, referred to as Type I PWM is the same as that of Fig. 9, and as discussed before, it uses 7212 instead of 0121 to reduce switching losses. Similarly, the scheme shown in Fig. $11 \mathrm{~b}$, referred to as Type II PWM, is obtained by modifying the five-zone PWM technique of Fig. 3b. From Fig. 5 and Fig. 6, it can be observed that 2721 results in low switching loss for the power factor angles considered, while 1012 results in switching loss that is even higher than the conventional sequence. Therefore, the Type II PWM uses 0127 in place of 1012 and 7212 in place of 0121 to achieve simultaneous THD and switching loss reduction.

The seven-zone hybrid PWM shown in Fig. 3c uses the 'bus-clamping' sequences 012 and 721 , at the mid- $\mathrm{V}_{\mathrm{REF}}$ range, to improve THD. These sequences involve only two switchings per sub cycle, and hence, the sampling frequency has to be increased by a factor of 1.5 , to maintain the same average switching frequency when these sequences are used. From Fig. 5, it can be seen that 721 results in lower switching losses, while 012 increases the switching losses. Further, there is not a significant difference in the THD performances of 012 and 721 in the $\mathrm{V}_{\mathrm{REF}}$ range considered. Therefore, Type I PWM can be modified to include 721 as shown in Fig. $11 \mathrm{c}$ and is referred to as Type III PWM. Finally, Type II PWM can be modified to include 721 resulting in Type IV PWM shown in Fig. 11d.
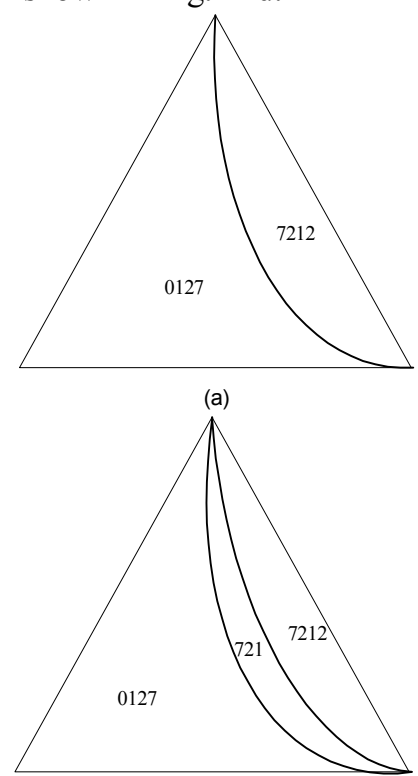

(c)

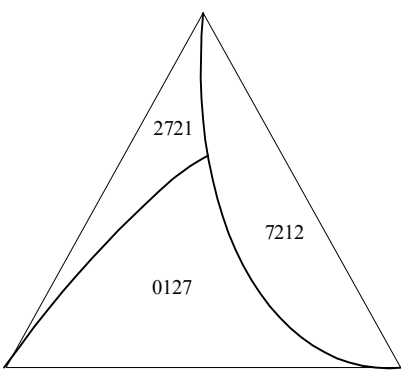

(b)

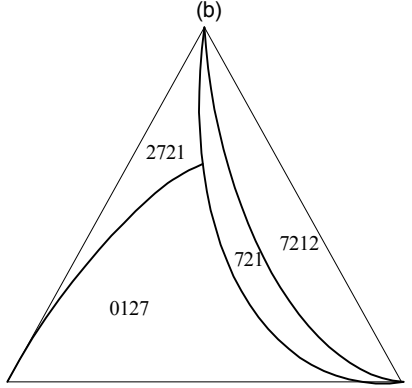

(d)
Fig. 11 Hybrid PWM techniques to reduce both THD and switching loss in induction motor drives

(a) Type I - 0127 and 7212, (b) Type II - 0127, 7212 and 2721, (c) Type III - 0127, 7212 and 721, (d) Type IV - 0127, 7212, 272 and 721 

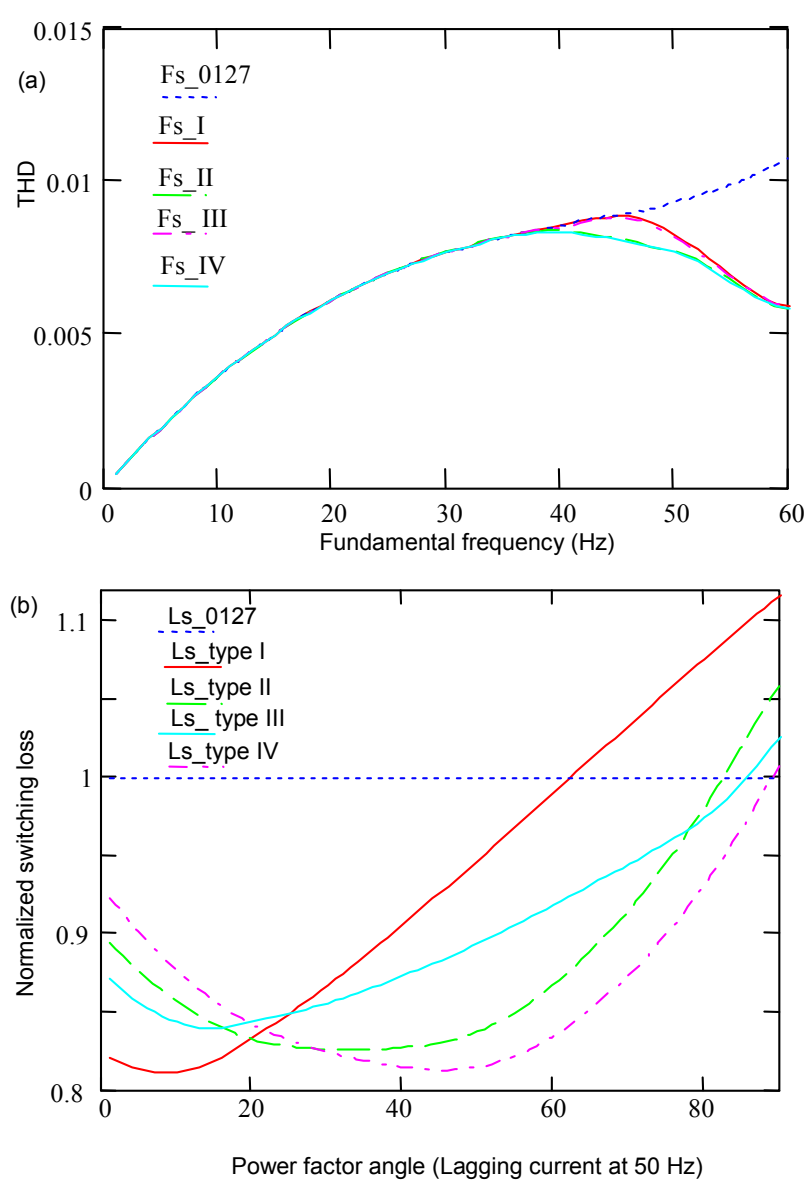

Fig. 12 THD and switching loss performance of combined hybrid PWM shown in Fig. 11, compared to conventional SVPWM

Analytical results comparing the THD and switching loss performance of the four proposed hybrid PWM techniques and the conventional SVPWM technique are shown in Fig. 12. It can be seen that introduction of sequence 721 results in significant improvement to THD performance in the fundamental frequency region from $40 \mathrm{~Hz}$ to $50 \mathrm{~Hz}$, as well as a significant reduction in switching losses. As seen in Fig. 11, at a higher fundamental frequency close to $60 \mathrm{~Hz}$, all the PWM techniques use 7212 through out the sector. Therefore, the performance of the four new PWM techniques will be similar at a fundamental frequency of $60 \mathrm{~Hz}$. However, at lower fundamental frequencies of $40 \mathrm{~Hz}-50 \mathrm{~Hz}$, the advantage of using 721 and 2721 to reduce switching losses can be clearly seen from Fig. 12b (corresponding to fundamental frequency of $50 \mathrm{~Hz}$ ). Finally, it can be observed from Fig. 12 that all the four proposed PWM techniques result in simultaneous reduction in THD and switching losses, compared to the conventional SVPWM, for power factor angles in the range of $20^{\circ}$ to $60^{\circ}$ lagging.

\section{EXPERIMENTAL RESULTS}

The characteristics of different sequences have been studied experimentally on an IGBT based inverter driving a three-phase, 2hp induction motor. The proposed PWM techniques as well as the conventional SVPWM are implemented using a digital signal processor, TMS320F243 from Texas Instruments with a CPU clock frequency of 20 MHz. Fig. 13(a) shows the PWM waveforms corresponding to phase ' $a$ ', along with phase 'a' current for conventional sequence 0127. Fig. 13(b) and (c) show the corresponding waveforms for the switching-loss-optimized hybrid PWM suggested in Fig. 6, at two power factor angles $30^{\circ}$ and $60^{\circ}$ lagging. As seen, PWM using 2721 and 2721 results in clamping or single switching of phase 'a' around the peak of the current and double switching near the zero crossing of the current, thereby reducing the switching losses compared to conventional SVPWM.

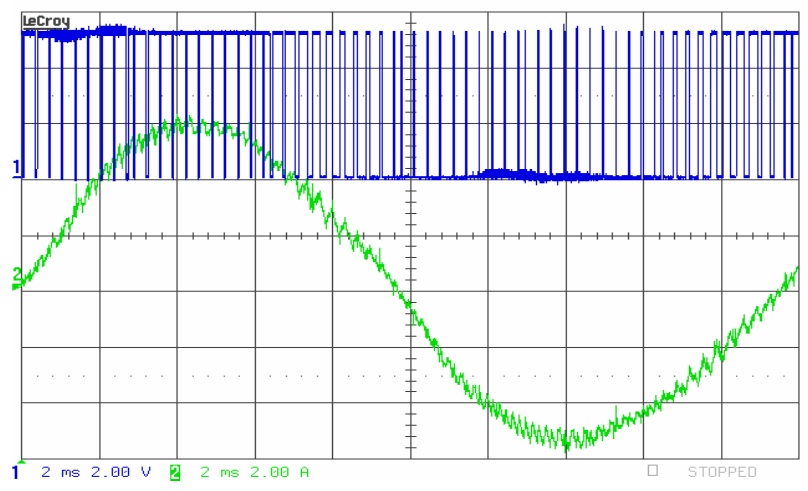

(a) conventional sequence 0127

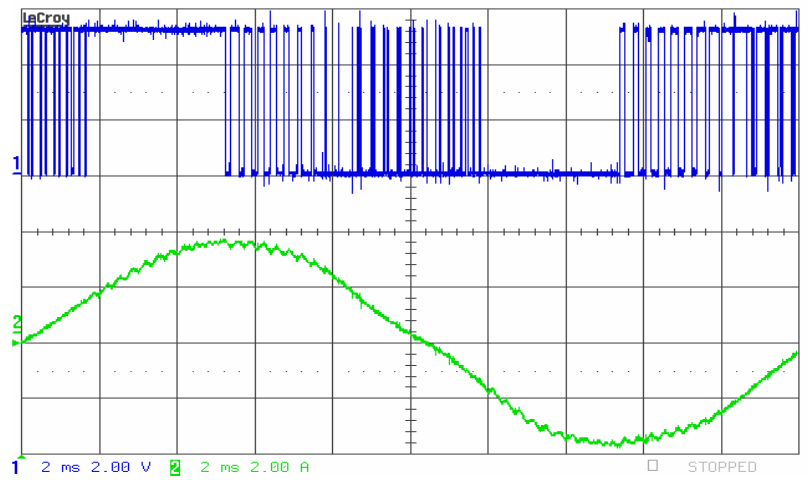

(b) Hybrid technique considering only switching loss performance as indicated by Fig. 6 (30 degree lagging current)

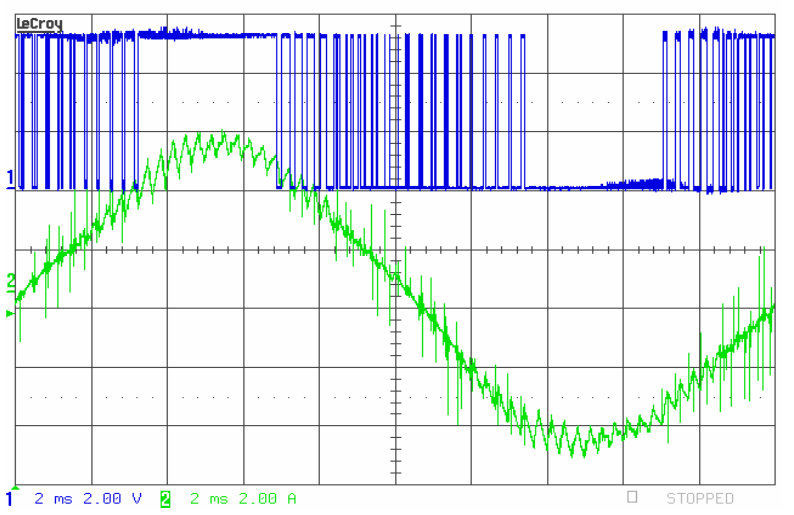

(c) Hybrid technique considering only switching loss performance as indicated by Fig. 6 (60 degree lagging current)

Fig. 13 Experimental PWM waveforms and motor currents of switching loss optimized PWM technique corresponding to phase a, compared to conventional PWM (fundamental frequency applied is $50 \mathrm{~Hz}$ ) 


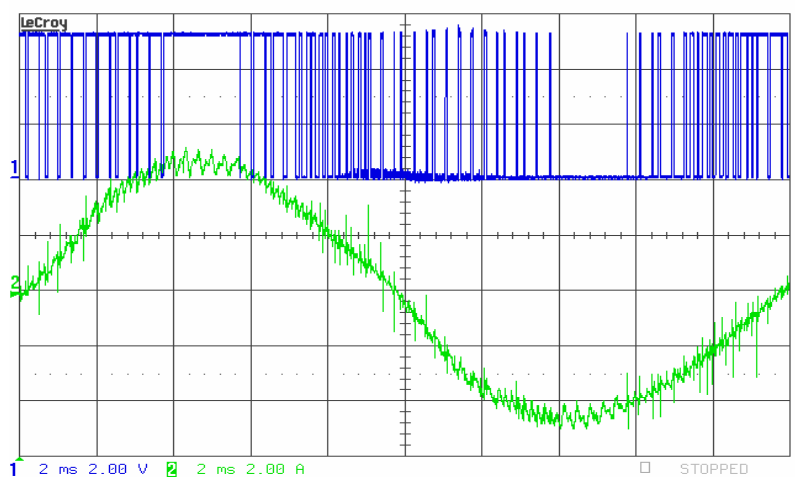

(a) hybrid PWM technique type I indicated by Fig. 11 (60 degree lagging current)

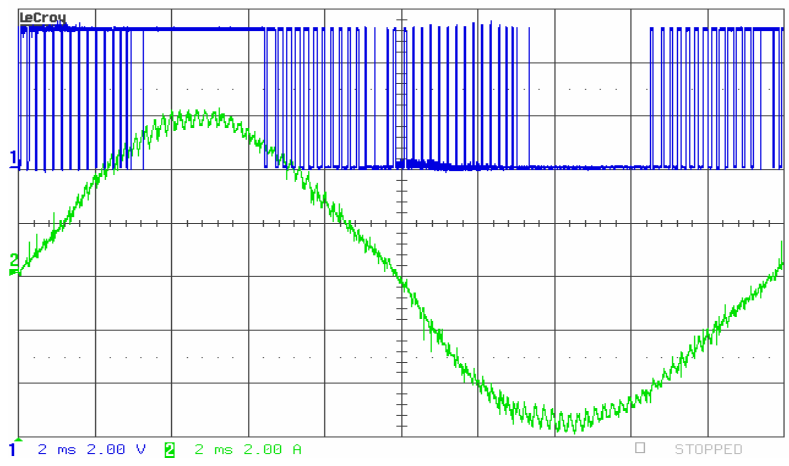

(b) hybrid PWM technique type IV indicated by Fig. 11

Fig. 14 Experimental PWM waveforms and motor currents of hybrid PWM techniques to reduce both switching loss and THD of motor current corresponding to phase a (fundamental frequency applied is $50 \mathrm{~Hz}$ )

Direct measurement of switching losses by measuring the instantaneous switch current and switch voltage is not practical. Finite differences in the delay times of voltage probes and current probes introduce a large error in power loss measurements, especially considering that the total switching losses, and therefore the savings achieved by the proposed schemes are small. Besides with an integrated three-phase IGBT module, the terminals are also not easily accessible for current measurements. An alternate approach used here is to compare the temperature rise of the heatsink on which the IGBT is mounted, for different PWM techniques. The temperature rise depends on the total power loss in all the three phases, and includes both conduction losses as well as switching losses. The conduction losses are independent of the PWM technique used. In order to obtain the conduction losses and switching losses separately, the system is operated at two different switching frequencies keeping all other parameters such as dc bus voltage, fundamental frequency and load unchanged. For example, when the switching frequency is doubled, the conduction losses remain the same, and the switching losses are doubled. Therefore, the difference in temperature rise between the two cases corresponds to the temperature rise due to switching losses alone, at the original switching frequency. The temperature rise due to switching losses alone for the conventional SVPWM, corresponding to the waveforms shown in Fig. $13 \mathrm{a}$ is $2.6{ }^{0} \mathrm{C}$ where as the temperature rise due to switching losses using the switching-loss-optimized PWM, corresponding to the waveforms shown in Fig. $13 \mathrm{c}$ is $2.0^{\circ} \mathrm{C}$. Therefore, the new hybrid PWM technique reduces the switching losses by $30 \%$ compared to the conventional SVPWM.

Fig. 14(a) and (b) show the PWM waveforms and the line currents of phase ' $a$ ' corresponding to the proposed PWM techniques Type I and IV suggested in Fig. 11, at a power factor angle of $30^{\circ}$ lagging. As seen, clamping or single switching at high current magnitudes and double switching near the zero crossing is achieved to ensure low switching loss, and low THD. An FFT (Fast Fourier Transform) analysis of the phase current waveform corresponding to Type IV PWM shows a 6\% reduction in THD compared to that of conventional SVPWM. (A reduction of $12 \%$ is expected from analysis and simulation).

\section{CONCLUSIONS}

New sequences involving division of active state time fully exploit the advantages of space vector based PWM compared to triangle comparison methods, and can result in simultaneous reduction in THD and switching losses. This paper analyzes the effect of the different sequences on the inverter switching losses. Based on the switching loss factor, $\mathrm{Q}$, defined in a sub-cycle, the $\alpha-\phi$ plane is divided into zones of superior performance for the different sequences in terms of switching loss. This can be used to design hybrid PWM techniques that will result in minimum switching losses.

By analyzing the switching losses characteristics along with the THD performance of the different sequences, four new hybrid PWM techniques that result in simultaneous reduction in THD and switching losses have been proposed, and experimentally validated. These techniques do not require line current feedback, and are simple to implement. These are meant for induction motor drive applications where the power factor angles are typically in the range of $20^{\circ}$ to $60^{\circ}$ lagging. However, the analysis can be extended to other applications, and new PWM techniques corresponding to different power factor angles can be easily derived. Finally, even better performance, in terms of both THD and switching losses, can be achieved if the hybrid PWM techniques are designed based on line current (or power factor) feedback.

\section{REFERENCES:}

[1] S. Ogasawara, H. Akagi and A. Nabae, "A novel PWM scheme of voltage source inverters based on space vector theory," $3^{\text {rd }}$ European Conf on Power Electronics and Applications, EPE'89, pp. 1197-1202, Oct 1989.

[2] J.Holtz, "Pulsewidth modulation - a survey," IEEE Trans. Industrial Electronics, vol. 39(5), pp.410-420, 1992.

[3] A.M.Hava, R.J.Kerkman, T.A.Lipo, "Simple analytical and graphical methods for carrier-based PWM-VSI drives," IEEE Trans. Power Electronics, vol. 14(1), 1999, pp. 49-61.

[4] H. Krishnamurthy, G. Narayanan, R. Ayyanar, V.T. Ranganathan, "Design of space vector based hybrid PWM techniques for reduced current ripple," IEEE APEC 2003, vol. 1, pp. 583-588.

[5] G.Narayanan, V.T.Ranganathan, "Synchronised PWM strategies based on space vector approach. Part 1: Principles of waveform generation," IEE Proc.-Electr. Power Appl, vol. 146(3), 1999, pp. 267-275.

[6] Harish K. Krishnamurthy, "Space vector methods for ac drives to achieve high efficiency and superior waveform quality", M.S. thesis, Arizona State University, Apr. 2003. 